\title{
Imaging of Colloidal Gold Nanoparticle Using Atomic Force
}

\section{Microscope}

NurulSabihah Zakaria ${ }^{1, a^{*}}$, SitiRabizahMakhsin ${ }^{2, b}$, Azlan Abdul Aziz ${ }^{1,3, c,}$ Khairunisak A. Razak ${ }^{2,3, d}$.

${ }^{1}$ School of Physics, UniversitiSains Malaysia, 11800 Penang, Malaysia

${ }^{2}$ School of Materials and Mineral Resources Engineering, UniversitiSains Malaysia, 14300 NibongTebal, Penang, Malaysia

${ }^{3}$ NanoBiotechnology Research and Innovation, INFORMM, UniversitiSains Malaysia, 11800Penang, Malaysia

Email: *anurulsabihah87@gmail.com (Corresponding Author), bct_rabizah@yahoo.com, 'lan@usm.my, ${ }^{\mathrm{d}}$ khairunisak@eng.usm.my

Keywords: Nanotechnology, gold nanoparticle, imaging, atomic force microscopy.

Abstract. Colloidal gold is a suspension of sub-micrometer-sized particle of gold in a fluid usually water. The liquid is usually either an intense red color (for particle less than $100 \mathrm{~nm}$ ) or a dirty yellowish color (for larger particle). In this articles, the method of imaging using XE-BIO Atomic Force Microscopy (AFM) on gold nanoparticle (AuNP) synthesized by citrate reduction method have been developed to determine the size of the nanoparticle even when they are agglomerated. The spherical size of $20 \mathrm{~nm}, 30 \mathrm{~nm}$ and $40 \mathrm{~nm}$ gold nanoparticle can be measured using AFM images through vertical height measurement by analyzing a number of separate particles individually lying on the flat surface. It has been demonstrated that AFM gives the fastest way to determine the size, analyzing hundred of nanoparticles and produced images in all three dimensions compared to Transmission Electron Microscopy (TEM). 


\section{Introduction}

Colloidal gold or also known as gold nanoparticle (AuNP) is a suspension of sub-nanometer-sized particle of gold in a fluid usually water. The synthesized AuNP have particle sizes ranging from, e.g. $10 \mathrm{~nm}$ to $100 \mathrm{~nm}$ with color changing from an intense red color (for particle less than $100 \mathrm{~nm}$ ) to a dirty yellowish color (for larger particle). The size of AuNP determines their unique optic, electronic and magnetic properties. AuNP nowadays has widely used in material science [1] and biomedical $[2,3]$. For many of this application, the AuNP need to be monodispersed and have a specific size. Generally, colloidal AuNP can be synthesis as monodispersed nanoparticles with core sizes ranging from $1 \mathrm{~nm}$ to $250 \mathrm{~nm}$. The synthesis of AuNP can be controlled in different size and shapes due to their ability to react and agglomerate with other nanoparticles in their ambient condition [3]. Furthermore, AuNP can also becomes more prone to flocculation and aggregation [4]. As the size of colloidal AuNP increase so do their sensitivity to salt and environment. AuNP have increasingly gain interest due to their unique properties ofcontrolable morphology [5] and size dispersion [6,7], less toxicity and ease in synthesis and detection.

Since the invention of Atomic Force Microscopy (AFM) [8], amazing progress has rapidly growing in using AFM as powerful tool in science and technology research and development and become one of the most important instrument in colloid and interface science. The AFM is rather different from other microscope because it does not form an image by focusing light or electrons on a surface like optical or electron microscope. An AFM physically 'feels' the sample surface (scanning a probe over sample surface) with a sharp tip, building a map of the height of the sample surface. Besides, AFM is normally a surface investigation that not only gives the information about topographical but enable to deliver multiple information on material properties such as electrical, mechanical and chemical characterization when the tip make a contact with the sample surface. AFM is able to measured high resolution three-dimensional topography image from angstrom level to the micron scale. 
In general, there are three methods to study the characterization of nanoparticle including AFM, Transmission Electron Microscopy (TEM) and Scanning Electron Microscopy (SEM). However, TEM is very well known for very time consuming and exhibits the greatest constraint with regards to sample preparation while SEM are easier to prepare but required conductivity sample [9]. In contrast, AFM give major advantages because it is quicker and does not required a specific sample preparationbut yields data that are at least straightforward and easy to analysis [10]. Besides, the sample does not have to be conductive and no requirement for operation within a vacuum. Furthermore, AFM imaging requires the particle to be rigidly anchoring to a substrate.AuNP are very sensitive to their environment factors such as $\mathrm{pH}$, temperature, electrolyte and solvent. So, for these AuNP to be rigidly attached to the surface, a key issue is their surface chemistry and functionalization that determine the stability, functionality and application. Hence, to solve this issue, 3-aminopropyldimethylethoxysilane (APDMES) are being used to functionalized the sample surface by facilitate the chemical bonding between the particle and surface for immobilization of nanoparticle.Functionalized surfaces might either promote absorption or allow covalent bonding between the particle and substrate. So, particle allowed for greater reagent mobility and more complete surface coverage.

\section{Experimental Section}

Preparation of AuNP. General protocol for synthesis and storage of AuNP have been described by [11] with slightly modification [12]. The 20, 30 and $40 \mathrm{~nm}$ of AuNPs was synthesized using the citrate reduction method. Briefly, $0.01 \%(\mathrm{w} / \mathrm{v})$ of $\mathrm{HAuCl}_{4} .3 \mathrm{H}_{2} \mathrm{O}$ was prepared in an Erlenmeyer flask, then stirred and heated to boiling temperature. Then, $1 \%(\mathrm{w} / \mathrm{v})$ trisodium citrate was added rapidly. The solution was further boiled for 10 minutes to ensure complete reaction took place. The amount of trisodium citrate added was attuned from $2 \mathrm{ml}, 1.4 \mathrm{ml}$ and $1 \mathrm{ml}$ to produce 20,30 and 40 nm colloidal AuNPs, respectively. The solution was stirred constantly for several minutes and then cooled to room temperature. The suspension was then stored at $4{ }^{\circ} \mathrm{C}$. 
Deposition of AuNP. The gold deposition procedure can be conducted using silicon as the substrate material. A small sample ( $5 \mathrm{~mm}$ x $5 \mathrm{~mm})$ was cleaved from a silicon wafer. The sample was cleaned through RCA cleaningmethod. The wafer than rinsed with deionized water and blown dry with high purity nitrogen. The deposition of AuNP on silicon surface has been carried out in two steps; first method is using a bare silicon substrate and the second method is using modified-APDMES silicon substrate. Firstly, $25 \mu \mathrm{L}$ of AuNP $(20 \mathrm{~nm}, 30 \mathrm{~nm}$ and $40 \mathrm{~nm})$ applied into silicon substrate using micropipette. The gold solution should spread evenly across the surface. In order to dry this sample, they areheated at $200{ }^{\circ} \mathrm{C}$ for 1 hour in an oven. Secondly, the silicon substrate being modified by 3aminopropyldimethylethoxysilane (APDMES) (Sigma-Aldrich) [13] for immobilization of nanoparticle. For the silanization, $5 \mu \mathrm{ml}$ of APDMES placed on the Si substrate. The APDMES was allowed to react with the substrate for 2 hours inside a petri dish. The excess of APDMES then rinsed off with ethanol followed by deionized water. After drying, $25 \mu \mathrm{L}$ of AuNP applied into the APDMES-modified silicon substrate using micropipette. The substrate was then incubated at room temperature. For $20 \mathrm{~nm}$ AuNP incubation time was $20 \mathrm{~min}$, followed by $30 \mathrm{~nm}$ for $30 \mathrm{~min}$ and 40 $\mathrm{nm}$ for $40 \mathrm{~min}$. The incubation time varied with the size of the nanoparticle to modify the particle density on the silicon surface. Known that, gold display higher particle density and independent on the pretreatment of the silicon substrate and surface condition of silicon. Size of deposited particle decreased from $100 \mathrm{~nm}$ to $10 \mathrm{~nm}$ as the particle density increased. After that, the sample rinsed with ethanol followed by deionized water, and dry with dry nitrogen priorto analysis.

AFM Imaging. Imaging was carried out using XE-BIO AFM (Parks Systems) in the AFM noncontact mode of operation in air. Silicon nitride tips with a nominal $2 \mathrm{~nm}$ radius, $125 \mu \mathrm{m}$ of length,force constant of $42 \mathrm{~N} / \mathrm{m}$ and a resonance frequency of about $330 \mathrm{~Hz}$ were used. The approach-retract cycle were carried out at typical z-scan rate of $0.1 \mathrm{~Hz}$ to $1 \mathrm{~Hz}$. The imaging size was $2 \times 2 \mu \mathrm{m}^{2}$ unless otherwise specified. 


\section{Result and Discussion}

Operation of AFM. In order to obtain accurate image of nanoparticle using AFM, they must first be attached to a flat surface such as silicon, mica or glass slide. All AFM images were taken using noncontact mode with PPP-NCHR cantilever. The specification of the cantilever and sharp tip plays an important role in determining the sensitivity and resolution of the AFM. A topographic AFM image (Fig. 1, Fig. 2) is actually a convolution of the tip and sample geometry. However, this not affect the height of the measurement that was used to determine the size of nanoparticle but it effect the overall surface features. To minimize the convolution, the tip with radii $<10 \mathrm{~nm}$ is the best to be used. The cantilever with stiffness in approximation to $40 \mathrm{~N} / \mathrm{m}$ are recommended to used to overcome the adhesive force (van der Waals and electrostatic forces) between the tip and sample [13]. In this paper, the cantilever with radius of $2 \mathrm{~nm}$ and stiffness of $42 \mathrm{~N} / \mathrm{m}$ was used.

AFM images have lateral $(\mathrm{x}, \mathrm{y})$ resolution and vertical $(\mathrm{z})$ resolution. The number of data point present in the $\mathrm{x}$ and $\mathrm{y}$ scan direction on image needs to be taken into consideration during image analysis. It is important to consider the particle size first when choosing the scan size. At first, used a large scan size $\left(30 \times 30 \mu \mathrm{m}^{2}\right)$ to identify a region with a homogenous particle distribution. Once a suitable scan area $\left(2 \times 2 \mu \mathrm{m}^{2}\right)$ has been decided, the scanning of image will start. Adjust the oscillation amplitude feedback gain by playing over the control panel such as set point, scan rate and z servo gain until the forward and backward line scan fit to each other (identical) that shown the signal is good. Prior analysis, the first step in AFM image processing is all imaged need to be go through flattening technique using a polynomial fit by fitting each scan line and subtracting it from the data to remove the artifact from the image acquisition process [14]. A first regression order (linear) correction is normally enough to remove any artifact. 

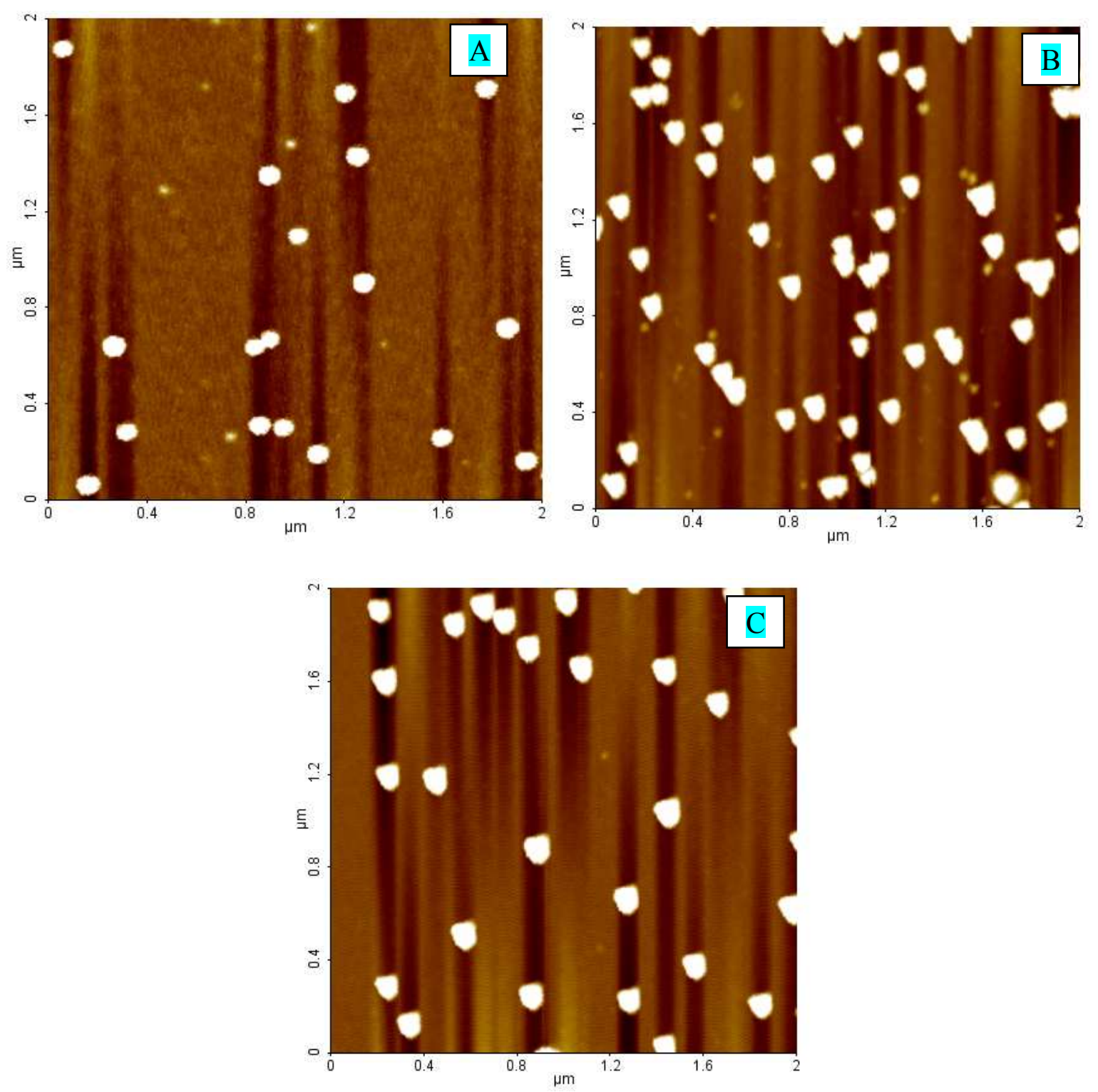

Fig. 1. A $2 \times 2 \mu \mathrm{m}^{2}$ non-contact mode AFM topographical image of (A) $20 \mathrm{~nm}$ AuNP (B) $30 \mathrm{~nm}$ AuNP and (C) $40 \mathrm{~nm}$ AuNP with modified APDMES-Silicon substrate after flattening technique. 

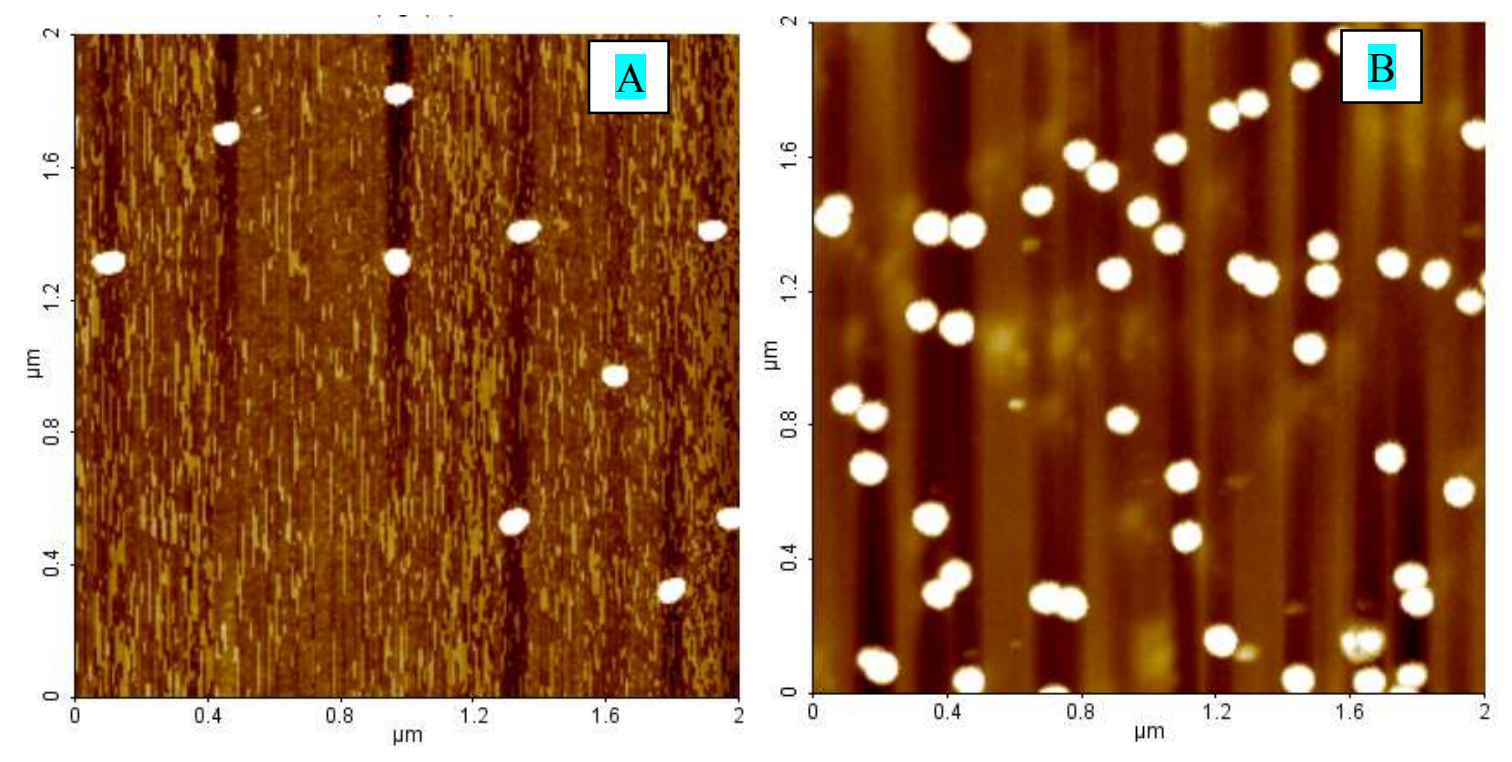

Fig. 2. A $2 \times 2 \mu \mathrm{m}^{2}$ non-contact mode AFM topographical images of silicon surface covered by (A) 30 nm AuNP and (B) $40 \mathrm{~nm}$ AuNP.

Size measurement. Fig. 1 shows a non-contact AFM topography image and particle distribution of a APDMES-coated silicon surface covered with $20 \mathrm{~nm}, 30 \mathrm{~nm}$ and $40 \mathrm{~nm}$ AuNP synthesis bycitrate reduction methodof [12] as the reference system while Fig. 2 shows AFM image of AuNP deposited onto the bare silicon substrate. The surface was scanned underambient air condition. The image shows that the AuNP are approximately in a spherical shape. The size measurement of the nanoparticle wasobtained by measuring the vertical height of the particle $[14,15]$. By making several cross-sectional line profiles through nanoparticle, the particle height can be determined by the difference between the peak height and the baseline from both side of the nanoparticle. Furthermore, the particle individually separation and accuratelylying on a flat region also can be obtained. Park System developed the Crosstalk Elimination (XE) AFM based on decoupled flexure scanners where the XY scanner only moves the sample and the $\mathrm{Z}$ scanner drives the probe. The XE-BIO AFM removes the scanner bowing, hence attaining flat XY scan and improves the Z-servo response, resulting in superb tip preservation by non-contact mode. 
Fig. 3 shows that a uniform gold nanoparticle layer formed on the silicon surfaced. The average measurement of particle height from the AFM image yield a value of $30 \pm 1 \mathrm{~nm}$, while in Fig. 4, the average particle height is about $40 \pm 2 \mathrm{~nm}$, well consistent with TEM result (Fig. 8). In the case of the spherical nanoparticles, the AFM only scan the upwards facing surface of the particle as a topography image. The observed width/height ratio is always greater than 1 . This is due to the finite radius of the AFM tip result in the broadening of the particle and the flattening process of particulate objects when absorbed to a surface [15]. Therefore, assuming that the nanoparticle is in a spherical shape. The particle in Fig. 2(A) shown that it does not dispersed evenly compared to Fig. 1(B) that the surface was functionalized with APDMES. Functionalized surfaces might either promote absorption or allow covalent bonding between the particle and substrate and also enhanced the surface interaction.So, particle allowed for greater reagent mobility and more complete surface coverage [16].
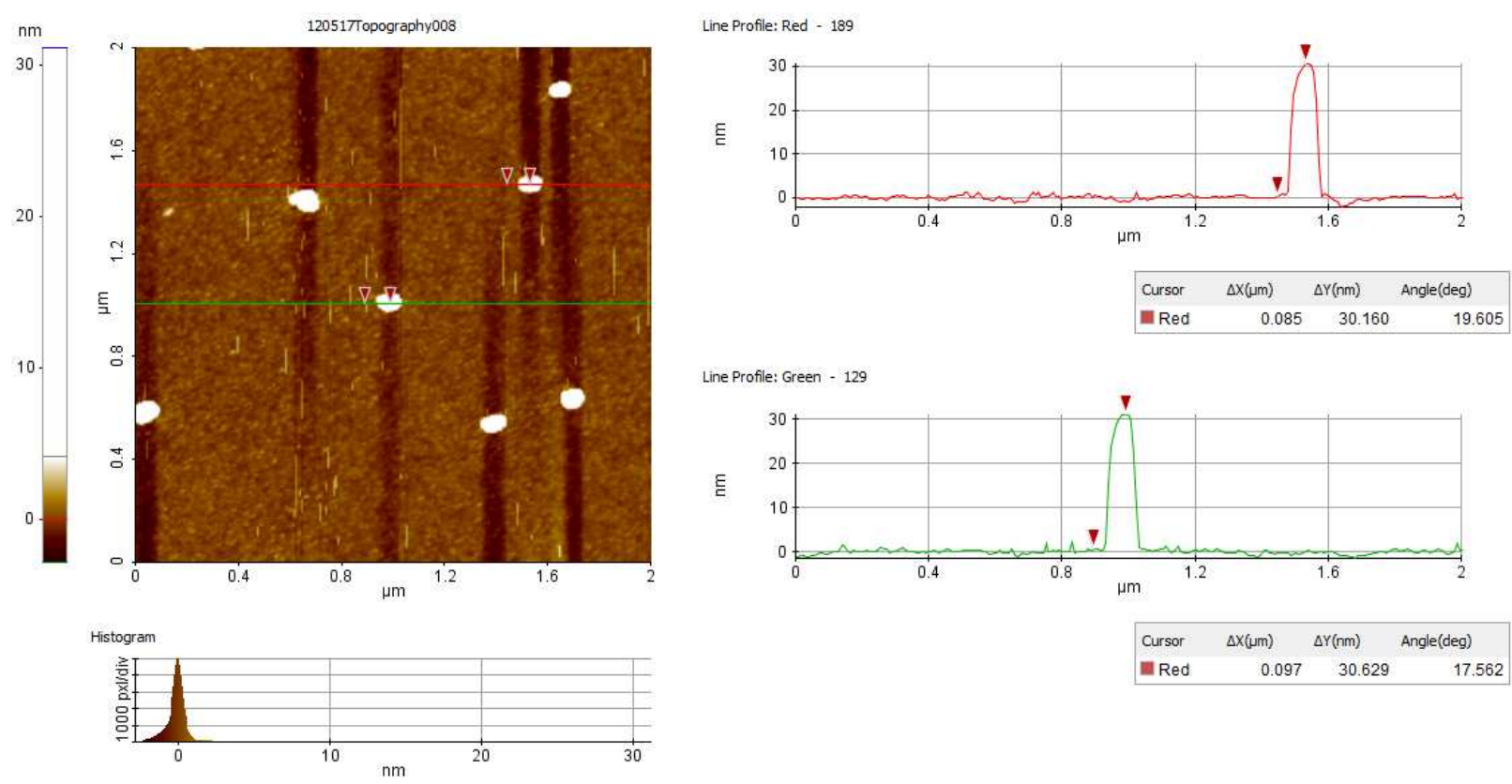

Fig. 3. Cross-sectional line profile for $30 \mathrm{~nm}$ AuNP. 

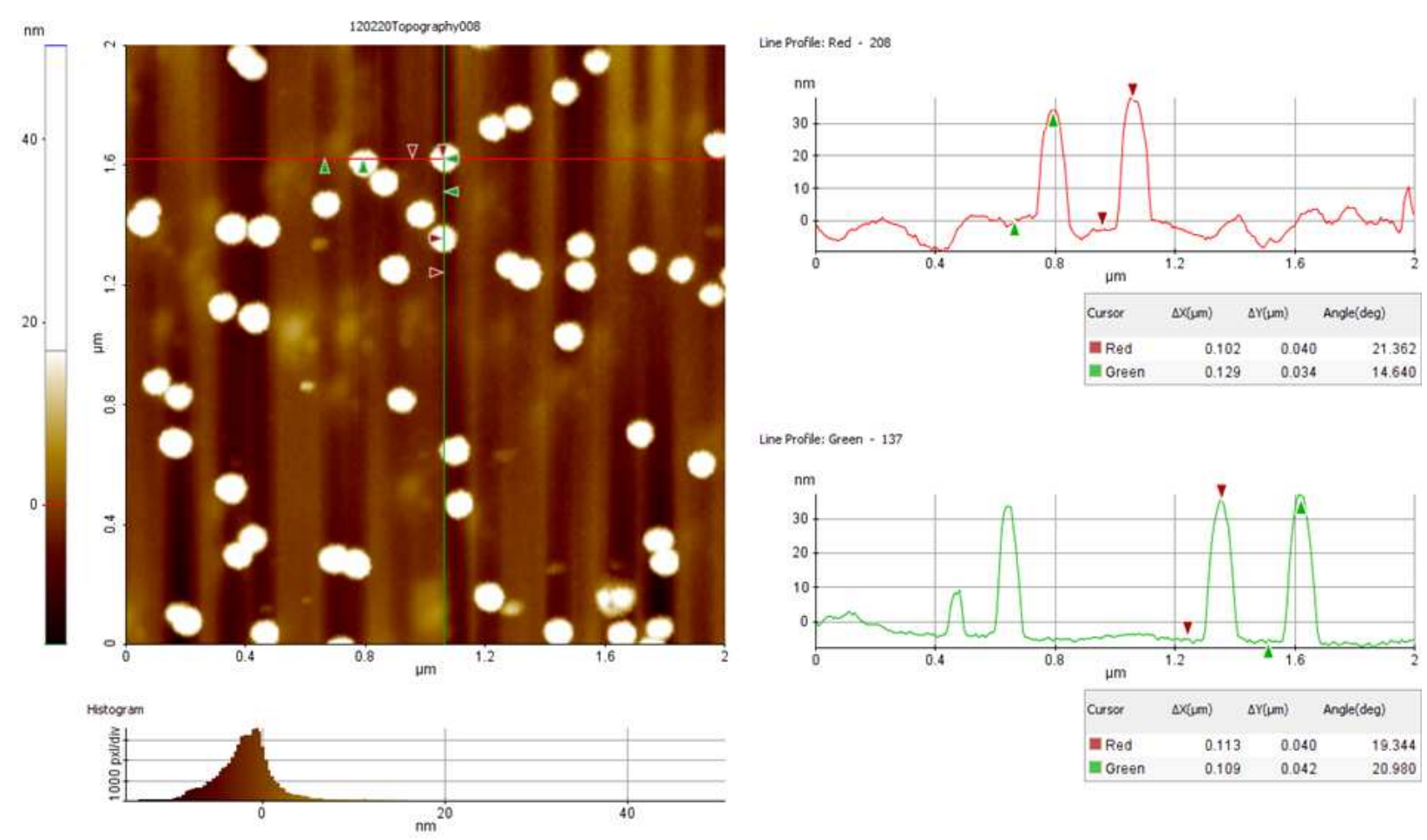

Line Profile: Green - 13 ?

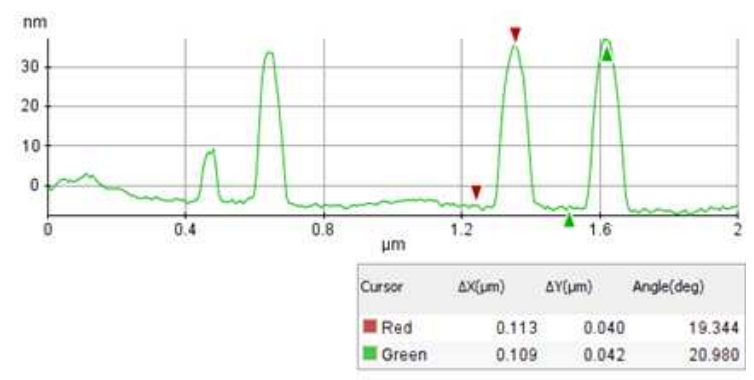

Fig. 4. Cross-sectional line profile for $40 \mathrm{~nm}$ AuNP.

Fig. 5, 6 and 7 shows the cross-sectional profile line for $20 \mathrm{~nm}, 30 \mathrm{~nm}$ and $40 \mathrm{~nm}$ AuNP respectively with modified APDMES-Silicon surface.APDMES is one of the aminosilane reagents that are generally used for functionalization of silicon surface and immobilization of nanoparticle. The two methyl groups on APDMES contribute some steric hindrances that give adsorbed substrate some measure of hydrophobility. Furthermore, APDMES has one anchoring point to the surface or other silane molecules and simplified to dimerized [16]. It shown that the height of each particle is quite bigger compared to the height of particle in Fig. 3 and Fig 4. Consequently, the thickness layer of APDMES can be obtained. As can see that the thick layer coated the bright spot in the image. Random features such as this may be mistakenly believed as the size of AuNP because the image is totally based on topography. In general, for AFM analysis, the smaller the size of the particle, the smoother the substrate must be. For $30 \mathrm{~nm}$ AuNP (Fig. 3) shown that the value of rms roughness is lower (1.835) compared to the value for $40 \mathrm{~nm}$ AuNP (1.888) (Fig. 7). Furthermore, silanized surface also shown that the rms roughness of the surface is lower than the bare silicon surface. 
From the line profile, the average height for $30 \mathrm{~nm}$ AuNP coated with APDMES yield a result of $37 \pm 2 \mathrm{~nm}$. If compared to the line profile in Fig. 3, the result for AuNP itself is about $29 \pm 1 \mathrm{~nm}$. While for $40 \mathrm{~nm}$ AuNP, the result for coated APDMES is about $47 \pm 2 \mathrm{~nm}$ compared to AuNP in Fig. 4 is about $40 \pm 2 \mathrm{~nm}$. So, the thickness layer for APDMES coated with AuNP is obtained approximately $8 \mathrm{~nm}$. The image for $30 \mathrm{~nm}$ and $40 \mathrm{~nm}$ AuNP shows that the shape of the nanoparticle is not approximately spherical due to the effect of the tip encountered when the AFM tip scans features which has radii of curvature similar or smaller than the tip [17].
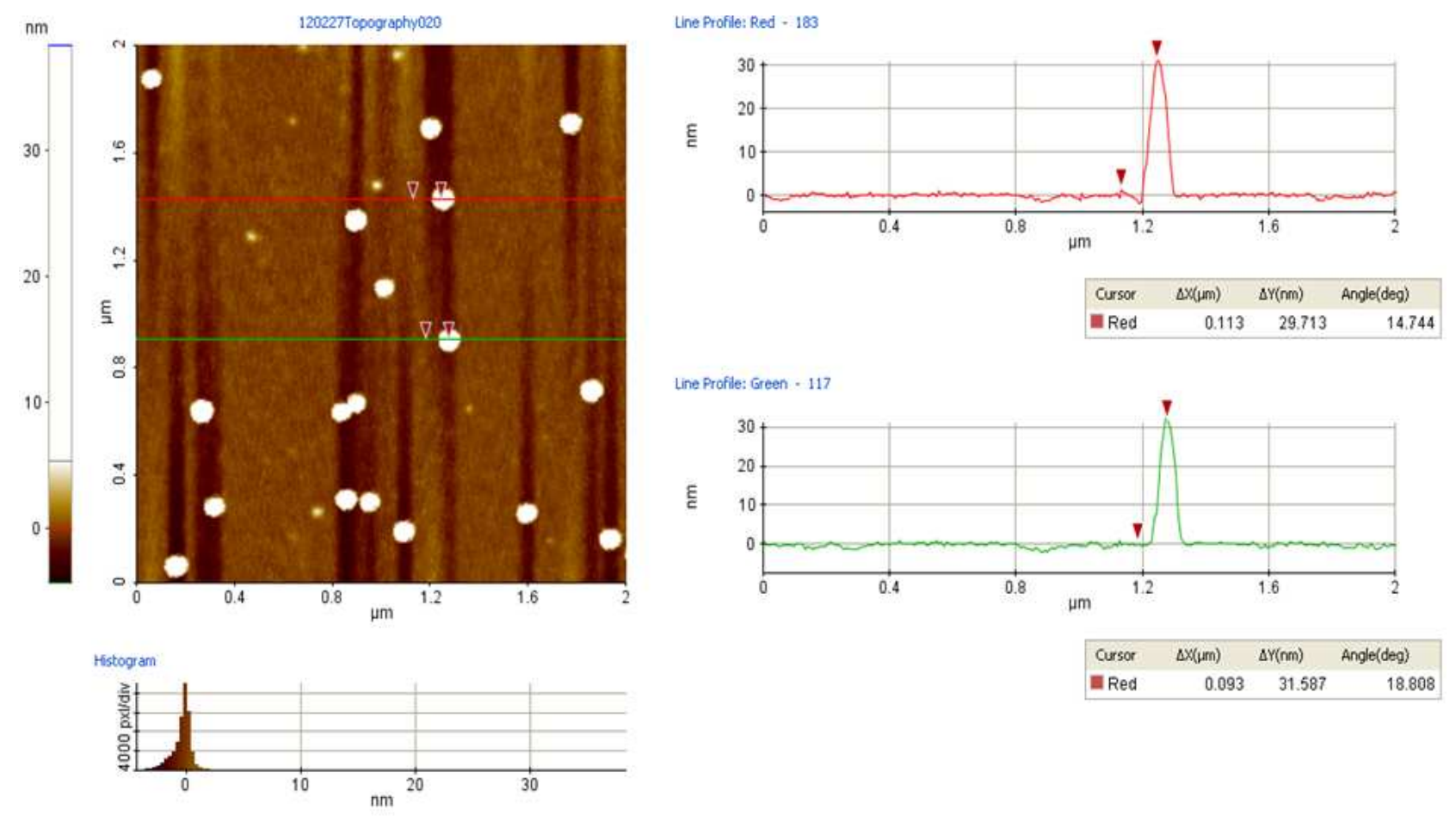

Fig. 5. Cross-sectional line profile for $20 \mathrm{~nm}$ AuNP with APDMES-modified silicon surface. 

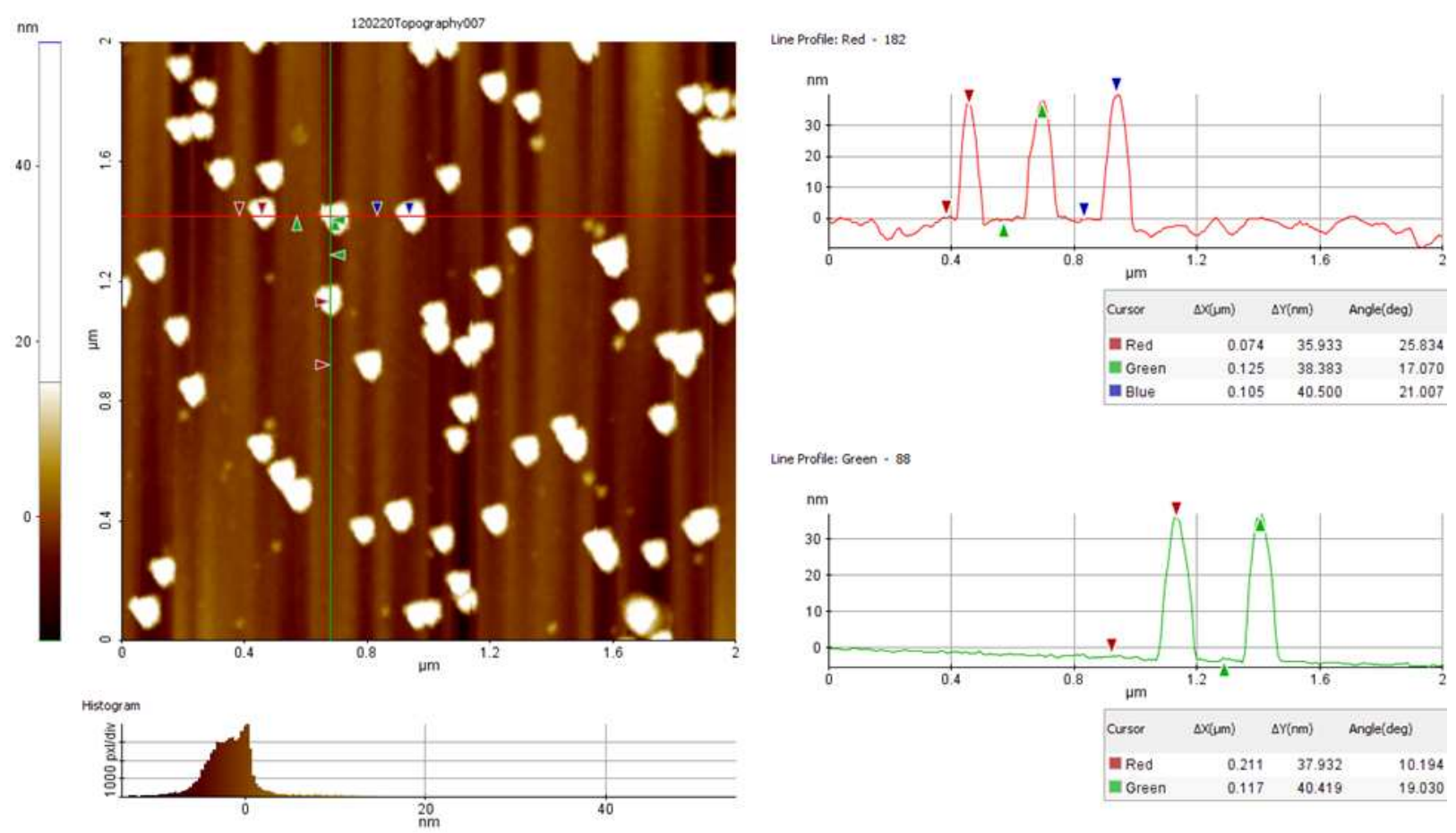

Fig. 6. Cross-sectional line profile for $30 \mathrm{~nm}$ AuNP with APDMES-modified silicon surface.
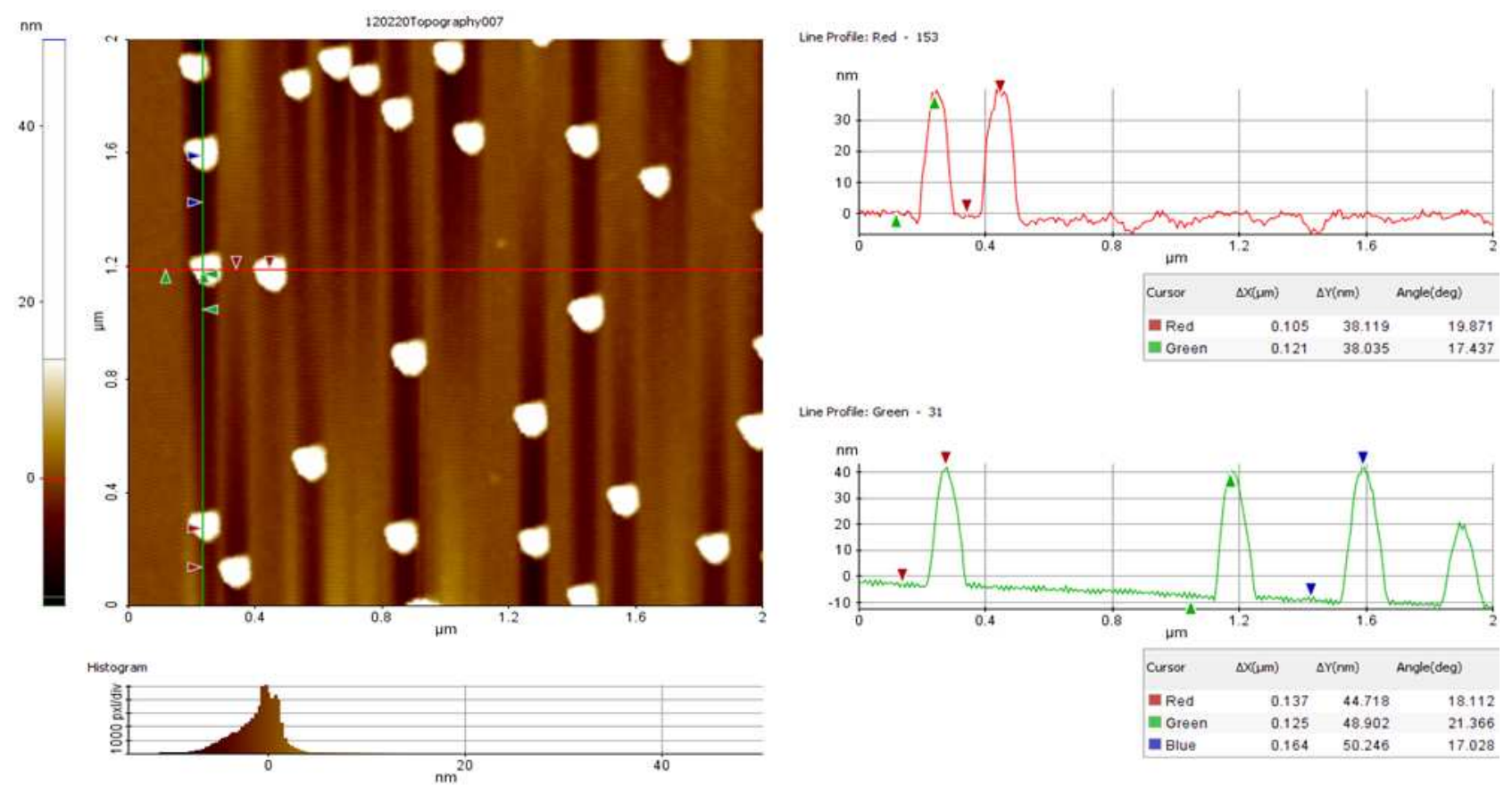

Fig.7. Cross-sectional line profile for $40 \mathrm{~nm}$ AuNP with APDMES-modified silicon surface. 


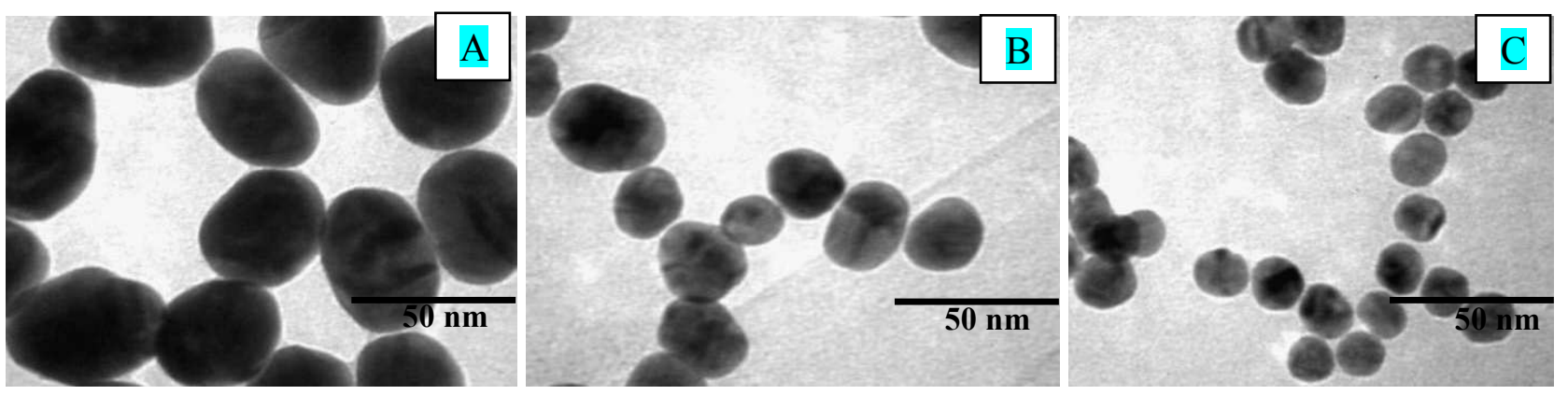

Fig. 8. TEM images of AuNPs with varied sizes (A) $40 \mathrm{~nm}$, (B) $30 \mathrm{~nm}$ and (C) $20 \mathrm{~nm}$.

\section{Conclusion}

The size measurement of the gold nanoparticle was obtained by measuring the vertical height of the particle of AFM image yield the value that well consistent to the TEM result. However, the measurement by AFM was relatively straightforward, ease in sample preparation and interpretation of data compared to TEM analysis. The silanized surface exhibiting lower rms roughness that provides reproducible immobilization of AuNP compared to bare silicon. So, particle allowed for greater reagent mobility and more complete surface coverage.

\section{Acknowledgement}

This research was supported by Research University grants 1001/PFIZIK/814113.

\section{Reference}

[1] M. Wanunu, R. Popovitz-Biro, H. Cohen, A. Vaskevich, I. Rubinstein, Coordination- based Gold Nanoparticle Layers, JACS 127 (25) (2005) 9207-15.

[2] W. Cai, T. Gao, H. Hong, Applications of Gold Nanoparticles in Cancer Nanotechnology, Nanotechnology, Science and Application (2008) 17-32.

[3] P. M. Tiwari, K. Vig, V.A. Dennis, S.R. Singh, Functionalized Gold Nanoparticles and Their Biomedical Applications, Nanomaterials 1(1) (2011) 31-63. (doi:10.3390/nano1010031) 
[4] S. Thobhani, S. Attree, R. Boyd, N. Kumarswami, J. Noble, M. Szymanski, R.A. Porter, Bioconjugation and Characterisation of Gold Colloid-labelled Proteins, J. Immunol. Meth. 356 (1-2) (2010) 60-9.

[5] S. Darwich, K. Mougin, A. Rao, E. Gnecco, S. Jayaraman, H. Haidara, Manipulation of Gold Colloidal Nanoparticles With Atomic Force Microscopy In Dynamic Mode: Influence of Particle-Substrate Chemistry And Morphology, And Of Operating Conditions, Beilstein J. Nanotechnol. 2 (2011) 85-98.

[6] K. Brown, D. Walter, Seeding of Colloidal Au Nanoparticle Solutions. Improved control of Particle Size and Shape, Chem. Mater. 12 (2) (2000) 306-313.

[7] N. Jana, L. Gearheart, Seeding Growth for Size Control of 5-40 nm Diameter Gold Nanoparticles, Langmuir 37 (2001) 6782-6786.

[8] G. Binnig, C.F. Quate, Atomic Force Microscope, Phys. Rev. Lett. 56 (1986) 930-933.

[9] N. Starostina, Part II: Sample Preparation for AFM Particle Characterization, Probe Microscopy, (2006).

[10] K.C. Grabar, K.R. Brown, C.D. Keating, S.J. Stranick, S. L. Tang, M. J. Natan, Nanoscale Characterization of Gold Colloid Monolayers: A Comparison of Four Techniques, Anal. Chem. 69 (3) (1997) 471-477.

[11] SR. Makhsin, KA. Razak, AA. Aziz, Study on Controlled Size, Shape and Dispersity of Gold Nanoparticles (AuNPs) Synthesized via Seeded-Growth Technique for Immunoassay Labeling, Adv. Mater. 364 (2012) 504-509.

[12] J. Grobelny, F. Delrio, N. Pradeep, D. Kim, NIST - NCL Joint Assay Protocol, PCC-6 Size Measurement of Nanoparticles Using Atomic Force Microscopy, National Institute of Standards and Technology, 21702, (2009).

[13] R. Boyd, New Analysis Procedure for Fast and Reliable Size Measurement of Nanoparticles from Atomic Force Microscopy Images, J. Nanopart. Res. 13 (1) (2011) 105-113. 
[14] J. Sitterberg, A. Özcetin, C. Ehrhardt, U. Bakowsky, Utilising Atomic Force Microscopy for the Characterisation of Nanoscale Drug Delivery Systems, Eur. J. Pharm. Biopharm. 74 (1) (2010) 2-13.

[15] F. Zhang, K. Sautter, A. M. Larsen, D.A. Findley, R.C. Davis, H. Samha, M.R. Linford, Chemical Vapor Deposition of Three Aminosilanes on Silicon Dioxide: Surface Characterization, Stability, Effects of Silane Concentration, and Cyanine Dye Adsorption, Langmuir 26 (18) (2010) 14648-14654.

[16] O. Couteau,G. Roebben, Measurement of The Size of Spherical Nanoparticles by Means of Atomic Force Microscopy, Meas. Sci. Technol. 22 (6) (2011). (doi:10.1088/09570233/22/6/065101). 